\title{
PSEUdo-SUPERSTRUCTURES AS Nonstandard UNIVERSES
}

\author{
Mauro Di Nasso
}

\begin{abstract}
A definition of nonstandard universe which gets over the limitation to the finite levels of the cumulative hierarchy is proposed. Though necessarily nonwellfounded, nonstandard universes are arranged in strata in the likeness of superstructures and allow a rank function taking linearly ordered values. Nonstandard universes are also constructed which model the whole ZFC theory without regularity and satisfy the $\kappa$-saturation property.
\end{abstract}

\section{Introduction.}

The traditional superstructure approach to the nonstandard methods is the one introduced by A. Robinson and E. Zakon in [RZ] and further developed in $[Z]$. It is based on the well-known fact that virtually all ordinary mathematics can be thought as embedded in set theory. Roughly speaking, it consists of a mapping between $\omega$-superstructures which provides a transfer for first order properties. Since then, other interesting approaches have been worked up: see $[\mathrm{N}],[\mathrm{HR}],[\mathrm{KA}],[\mathrm{FL}]$ and $[\mathrm{BH}]$. This paper is an attempt to generalize the superstructure approach by getting over the finite levels in the definition of standard model. In fact while $\omega$-superstructures are appropriate as a framework for calculus and real analysis, a much wider kind of a universe is needed in order to develop other mathematical theories, such as general topology or set theory.

Until recently the regularity axiom has been routinely included among the axioms of set theory. Thus, extending the notion of standard model was prevented as a consequence of the following fact. If the standard model satisfies the axiom of infinity, then the corresponding nonstandard universes 
are nonwellfounded. We point out that nonwellfounded sets are nowadays frequently used in order to arrange new theories in a set-theoretic framework. This is the case of foundational researches in mathematics, expecially self-descriptive set theories, and of various branches of theoretical computer science (see $[\mathrm{HI}],[\mathrm{A}]$ and $[\mathrm{AR}]$ for surveys on these subjects). All of these new aspects of mathematical research, more and more demand a set theory where a suitable anti-foundation axiom is adopted instead of regularity.

In their recent paper $[\mathrm{BH}]$, D. Ballard and K. Hrbacek take up this approach and construct nonstandard universes making use of Boffa's axiom of superuniversality. Their starting point is the notion of standard universe as any transitive proper class where all the relativizations of axioms of $Z F C$ set theory hold, with the exception of regularity. In this paper we are concentrating on the wellfounded sets as standard sets, and construct nonstandard universes which retain the main features of the superstructures. Though nonwellfounded, our nonstandard universes are full and transitive classes $\langle\mathbf{P}, \in\rangle$ provided with a rank function $\psi_{P}(p)=\operatorname{Sup}\left\{\psi_{P}\left(p^{\prime}\right)+1: p^{\prime} \in p\right\}$. Range $\left(\psi_{P}\right)=\lambda$ is a linearly ordered class which includes an increasing cofinal sequence indexed by the ordinals. In close analogy to the cumulative hierarchy, one can write $\mathbf{P}=\bigcup_{\xi \in \lambda} \mathbf{P}_{\xi}$ where $\mathbf{P}_{\xi+1}=\mathbf{P}_{\xi} \cup \mathcal{P}\left(\mathbf{P}_{\xi}\right)$ for every $\xi \in \lambda$. If the whole class $\mathbf{W F}$ of wellfounded sets is taken as standard universe, then the corresponding nonstandard universes model all $Z F C$ without regularity.

\section{Nonstandard Embeddings}

Throughout the paper we shall work in a nonwellfounded Zermelo-Fraenkel set theory with the axiom of choice and, instead of the axiom of regularity, a suitable anti-foundation principle. Precisely, the axioms of our set theory are those of $Z F C^{-}$:

EXT (Extensionality); Ø (Empty-set); PAIR (Pairing); SEP (Separation schema); $U N$ (Union); $P S$ (Power set); REP (Replacement schema); INF (Infinity); $A C$ (Choice)

and the following Boffa's axiom SU of superuniversality: 
Suppose that the extensional structure $\left\langle A^{\prime}, \epsilon^{\prime}\right\rangle$ is an end extension of $\langle A, \epsilon\rangle .{ }^{1}$ Then every isomorphism $\pi:\langle A, \epsilon\rangle \rightarrow\langle T, \epsilon\rangle$ onto a transitive $T$, can be extended to an isomorphism $\pi^{\prime}$ : $\left\langle A^{\prime}, \epsilon^{\prime}\right\rangle \rightarrow\left\langle T^{\prime}, \in\right\rangle$ onto a transitive $T^{\prime} \supseteq T$.

$S U$ states that a Mostowski transitive collapse is possible for every extensional binary structure, even if it is not wellfounded. Moreover, such an isomorphism can be extended to a transitive collapse for any of its end extensions. A proof of the relative consistency of $Z F C^{-}+S U$ can be found in $[\mathrm{B}]$. The reader interested in anti-foundation axioms is suggested to consult also $[\mathrm{FH}]$ and $[\mathrm{A}]$.

Further on in this paper, we shall mention the following modified version $E X T_{o}$ of the axiom of extensionality:

$$
\forall x \forall y[\exists t \in x \wedge \forall s(s \in x \leftrightarrow s \in y)] \rightarrow x=y
$$

In contrast to EXT, EXT allows a (possibly empty) class of atoms.

We shall use freely standard facts and notations of set theory and model theory from $[\mathrm{J}]$ and $[\mathrm{CK}]$, with the following particular conventions. Let $\mathcal{A}=$ $\langle A, \epsilon\rangle$ be a model for the language of set theory and $A^{\prime} \subseteq A$. For simplicity, throughout the paper we shall write $\left\langle A^{\prime}, \epsilon\right\rangle$ instead of $\left\langle A^{\prime}, \epsilon \cap\left(A^{\prime} \times A^{\prime}\right)\right\rangle$ to denote the submodel of $\mathcal{A}$ whose universe is $A^{\prime}$. We informally use classes in the style of $[\mathrm{J}]$. Except where explicitly noted, roman capital letters $A$, $B, C, \ldots$ will denote sets, while boldfaced letters $\mathbf{A}, \mathbf{B}, \mathbf{C}, \ldots$ will denote classes. ON will be the proper class of the ordinals and $\mathbf{W F}(X)=\bigcup_{\alpha} \mathrm{V}_{\alpha}(X)$ will be the wellfounded universe over $X$. Recall the cumulative hierarchy: $V_{o}(X)=X ; V_{\beta+1}(X)=V_{\beta}(X) \cup \mathcal{P}\left(V_{\beta}(X)\right) ; V_{\gamma}(X)=\bigcup_{\beta<\gamma} V_{\beta}(X)$ if $\gamma$ is limit. The sets of the form $\mathrm{V}_{\alpha}(X)$ are called $\alpha$-superstructures. A class $\mathbf{A}$ is transitive up to atoms if $a \in \mathbf{A}$ and $a \cap \mathbf{A} \neq \varnothing$ implies $a \subseteq \mathbf{A}$. A class $\mathbf{A}$ is full if $a \in \mathbf{A} \Rightarrow \mathcal{P}(a) \subseteq \mathbf{A}$. The notation $\mathcal{P}(A)$ for power-sets is extended to proper classes this way: $\mathcal{P}(\mathbf{A}) \doteq\{a \subseteq \mathbf{A}: a$ is a set $\}$.

The notion of standard model is usually grounded on a set $\mathrm{X}$ of individuals. For convenience, it is also assumed that such an $X$ consists of urelements.

\footnotetext{
${ }^{1}$ We remind the reader that $\left\langle A^{\prime}, \epsilon^{\prime}\right\rangle$ is an end extension of $\langle A, \epsilon\rangle$ if $A^{\prime} \supseteq A, a \epsilon b \Leftrightarrow a \epsilon^{\prime} b$ holds for all $a, b \in A$, and $a \epsilon^{\prime} b \in A \Rightarrow a \in A$. In other words, no member of $A$ gets a new element in $\left\langle A^{\prime}, \epsilon^{\prime}\right\rangle$. Observe that each $\langle A, \epsilon\rangle$ is trivially an end extension of the empty structure.
} 
However, as pointed out by J. Schmid and J. Schmidt in [SS], in order to apply nonstandard methods to various structures one should then claim that the number of urelements available exceeds each fixed cardinality. We prefer not to postulate the existence of a proper class of urelements (indeed the existence of any) for the sole purpose of nonstandard methods. As all one needs are elements which play the role of atoms, suitable sets will fit.

For any limit ordinal $\alpha$, call $X \alpha$-basal if $X$ is the set of atoms relative to $\left\langle\mathrm{V}_{\alpha}(X), \in\right\rangle$, i.e. if $\varnothing \notin X$ and $x \cap \mathrm{V}_{\alpha}(X)=\varnothing$ for each $x \in X$. Call $X$ basal if it is $\alpha$-basal for every $\alpha$. It is not difficult to obtain basal sets of any given size $\mu$.

Example. Consider $X=\left\{x_{j}: j \in \mu\right\}$ where $x_{j}=\left\{y_{j}\right\}, y_{j}=\left\{x_{j}, y_{j}\right\}$ and $x_{j} \neq y_{j^{\prime}}$ for every $j, j^{\prime} \in \mu$. Such $X$ 's are easily obtained by straight applications of the axiom $S U$.

A standard $\alpha$-model $\mathcal{S}$ is any $\in$-model of the form $\left\langle\mathrm{V}_{\alpha}(X), \in\right\rangle$ where $X$ is $\alpha$-basal. We now need a map $\vartheta$ to provide the fundamental transfer principle between the standard model and the nonstandard model. As shown in $[D]$, in those nonstandard universes which are elementary extensions, the internal sets make up a core with a strong rigidity property and the external collections form a mere frame which is completely useless for the sake of nonstandard methods. In particular, fundamental collections of internal sets such as the standard reals, the infinite reals, the monads, the galaxies and so on, are not in the model (see (ii) in the next proposition). This unpleasant fact suggests the opportunity of restricting the transfer principle to the bounded formulas. Recall that a formula $\sigma$ is bounded if every quantifier occurs in bounded form $\forall x(x \in y \rightarrow \ldots)$ or $\exists x(x \in y \wedge \ldots)$. Let $\mathcal{M}=\langle\mathbf{M}, \epsilon\rangle$ be a model of the language of set theory, with $\epsilon$ the $\mathcal{M}$ interpretation of the membership relation symbol. For each $m \in \mathbf{M}$, the collection $m_{\epsilon} \doteq\left\{m^{\prime} \in \mathbf{M}: m^{\prime} \epsilon m\right\}$ is assumed to be a set.

Definition We call the mapping $\vartheta:\left\langle\mathrm{V}_{\alpha}(X), \in\right\rangle \rightarrow\langle\mathbf{M}, \epsilon\rangle$ a nonstandard $\alpha$ embedding ( $\alpha$-NSE) when for every bounded formula $\sigma$ and every $a_{1}, \ldots, a_{n} \in$ $\mathrm{V}_{\alpha}(X)$ the following transfer principle holds:

$$
\left\langle\mathrm{V}_{\alpha}(X), \in\right\rangle \models \sigma\left(a_{1}, \ldots, a_{n}\right) \Longleftrightarrow \mathcal{M} \models \sigma\left(\vartheta\left(a_{1}\right), \ldots, \vartheta\left(a_{n}\right)\right)
$$

If $\mathcal{M}$ is an $\epsilon$-model, the additional property $\vartheta(\varnothing)=\varnothing$ is also required. We say $\mathcal{M}$ is a nonstandard $\alpha$-universe $(\alpha-N S U)$ relative to $\vartheta$. 
Notice that for every $A \in \mathrm{V}_{\alpha}(X) \backslash X, a \in A$ iff $\vartheta(a) \in \vartheta(A)$. Thus the image set $\vartheta[A] \doteq\{\vartheta(a): a \in A\} \subseteq \vartheta(A)_{\epsilon} \doteq\{m \in \mathbf{M}: m \in \vartheta(A)\}$. We shall consider proper NSE's only, that is embeddings where the above inclusion is proper for every infinite set $A$.

It is well-known that every NSE $\vartheta$ preserves the basic set-theoretic notions. E.g. for every $A, B \in \mathrm{V}_{\alpha}(X)$ the following hold: $A \subseteq B$ iff $\vartheta(A)_{\epsilon} \subseteq$ $\vartheta(B)_{\epsilon} ; \vartheta(A \cup B)_{\epsilon}=\vartheta(A)_{\epsilon} \cup \vartheta(B)_{\epsilon} ; \vartheta(A \cap B)_{\epsilon}=\vartheta(A)_{\epsilon} \cap \vartheta(B)_{\epsilon} ; \vartheta(A \backslash B)_{\epsilon}=$ $\vartheta(A)_{\epsilon} \backslash \vartheta(B)_{\epsilon}$. Moreover, every $n$-ary function $f: A^{n} \rightarrow B$ induces a unique $n$-ary function $f_{\vartheta}: \vartheta(A)_{\epsilon}^{n} \rightarrow \vartheta(B)_{\epsilon}$ such that $\left\langle\vartheta\left(a_{1}\right), \ldots, \vartheta\left(a_{n}\right)\right\rangle \mapsto$ $\vartheta\left(f\left(a_{1}, \ldots, a_{n}\right)\right)$. All these properties are proved by straight applications of the transfer principle to suitable bounded formulas (see $[\mathrm{Z}] \S 1$ ).

Definition An element $b \in \mathbf{M}$ is called $\vartheta$-standard if $b=\vartheta(a)$ for some $a \in \mathrm{V}_{\alpha}(X) ; \vartheta$-internal if $b \in \vartheta(a)$ for some $a \in \mathrm{V}_{\alpha}(X)$ and $\vartheta$-external if not internal.

The collection $\mathcal{I}_{\vartheta}=\bigcup_{\beta<\alpha} \vartheta\left(V_{\beta}(X)\right)_{\epsilon}$ of all the $\vartheta$-internal elements is $\epsilon$ transitive, i.e. $b^{\prime} \in b \in \mathcal{I}_{\vartheta}$ implies $b^{\prime} \in \mathcal{I}_{\vartheta}$. As the validity of bounded formulas is preserved under end extensions, the internal model $\left\langle\mathcal{I}_{\vartheta}, \epsilon\right\rangle \subseteq \mathcal{M}$ is an $\alpha$ NSU as well. We now itemize the main features of the special case $\alpha=\omega$.

1. Every internal model is wellfounded, thus it can be isomorphically collapsed onto an $\in$-structure which is transitive up to atoms. ${ }^{2}$

2. Up to isomorphisms, every internal model is the internal model of a NSE $*:\left\langle V_{\omega}(X), \in\right\rangle \rightarrow\left\langle V_{\omega}(Y), \in\right\rangle$ between $\omega$-superstructures. Thus, without loss of generality, one needs only consider $\omega$-superstructures as $\omega$-NSU's.

3. $\omega$-superstructures $V_{\omega}(Y)$ are transitive up to atoms and full.

4. $\omega$-superstructures $V_{\omega}(Y)$ are wellfounded; in particular they are provided with rank functions

$$
\rho_{Y}(A)= \begin{cases}0 & \text { if } A \in Y \text { or } A=\varnothing ; \\ \operatorname{Sup}\left\{\rho_{Y}(a)+1: a \in A\right\} & \text { otherwise. }\end{cases}
$$

\footnotetext{
${ }^{2}$ Here a slightly modified version of Mostowski's theorem applies which takes into account atoms.
} 
Properties 1, 3 and 4 are known facts. A proof of property 2 can be obtained as a consequence of 1 . We claim that the substance of the four properties above can be retained in our more general context $\alpha>\omega$, with the only exception of wellfoundedness. The next proposition states two basic facts about the internal sets.

\section{Proposition 1.1}

(i) The internal model $\left\langle\mathcal{I}_{\vartheta}, \epsilon\right\rangle$ is wellfounded iff $\alpha=\omega$. In particular, NSE's $\vartheta:\left\langle\mathrm{V}_{\alpha}(X), \in\right\rangle \rightarrow\left\langle\mathrm{V}_{\alpha}(Y), \in\right\rangle$ between $\alpha$-superstructures exist only when $\alpha=$ $\omega$.

(ii) Suppose the NSE $\vartheta:\left\langle\mathrm{V}_{\alpha}(X), \in\right\rangle \rightarrow\langle\mathbf{M}, \epsilon\rangle$ is an elementary embedding. If $\Lambda \in \mathbf{M}$ is a bounded collection of internal sets, i.e. if $\Lambda_{\epsilon} \subseteq \vartheta\left(V_{\beta}(X)\right)_{\epsilon}$ for some $\beta<\alpha$, then $\Lambda$ is internal.

Proof. (i) $V_{\omega}(X) \models \forall x^{\prime} \in x \in V_{n}(X) \rightarrow x^{\prime} \in V_{n-1}(X)$ for every natural number $n \geq 1$ and by transfer one gets that $\mathcal{I}_{\vartheta}=\bigcup_{n<\omega} \vartheta\left(V_{n}(X)\right)_{\epsilon}$ is wellfounded. Vice versa, suppose $\alpha>\omega$. In analogy to a well-known fact about the hypernaturals, $\epsilon$-descending chains exist for every $\xi \in \vartheta(\omega)_{\epsilon} \backslash \vartheta[\omega]$. (ii) Apply the transfer to $\mathrm{V}_{\alpha}(X) \models \forall x x \subseteq V_{\beta}(X) \rightarrow x \in V_{\beta+1}(X)$ (notice that such a formula is not bounded).

Wellfoundedness fails as soon as one goes beyond the finite levels of the cumulative hierarchy. We point out that, while this fact was crucial in the traditional set-theoretic approach, it has no effect within our anti-foundational framework. As for property (ii), it suggested our restricting the transfer principle to bounded formulas. In fact suppose the transfer holds for every formula. Then collections of internal sets which are routinely used in the practice of nonstandard methods (e.g. the infinitesimal numbers, the infinite natural numbers, the monads, the galaxies, etc.) would be unavailable in the NSU's.

Keisler's limit ultrapower construction yields an algebraic characterization for the internal models (basic results on limit ultrapowers can be found in $[\mathrm{CK}] \S 6.4)$. Let's fix our notations and denote $\left\langle\mathrm{V}_{\alpha}(X)_{D}^{I}, \varepsilon\right\rangle$ the ultrapower of $\left\langle\mathrm{V}_{\alpha}(X), \in\right\rangle$ modulo the ultrafilter $D$ over the set $I$. For every filter $F$ over $I \times I$, the corresponding limit ultrapower $\left\langle\mathrm{V}_{\alpha}(X)_{D}^{I} \mid F, \varepsilon\right\rangle \subseteq$ $\left\langle\mathrm{V}_{\alpha}(X)_{D}^{I}, \varepsilon\right\rangle$ is the submodel whose universe is made up by the $D$-equivalence classes $f^{D}$ of those functions $f: I \rightarrow \mathrm{V}_{\alpha}(X)$ which are almost constant: 
$\{\langle i, j\rangle \in I \times I: f(i)=f(j)\} \in F$. Notice that every ultrapower is a limit ultrapower (take $F=\mathcal{P}(I \times I)$ the trivial filter). We call $f^{D}$ bounded if $f^{D} \in V_{\beta}(X)_{D}^{I} \mid F$ for some $\beta<\alpha$ and we call bounded limit ultrapower the submodel $\left\langle{ }^{b} \mathrm{~V}_{\alpha}(X)_{D}^{I} \mid F, \varepsilon\right\rangle \subseteq\left\langle\mathrm{V}_{\alpha}(X)_{D}^{I} \mid F, \varepsilon\right\rangle$ made up by the bounded elements. Whenever confusion is unlikely, we shall identify each (bounded limit) ultrapower with its universe. It is easily seen that both the diagonal embedding $d: \mathrm{V}_{\alpha}(X) \rightarrow{ }^{b} \mathrm{~V}_{\alpha}(X)_{D}^{I} \mid F$ and the inclusion $\imath:{ }^{b} \mathrm{~V}_{\alpha}(X)_{D}^{I}\left|F \hookrightarrow \mathrm{V}_{\alpha}(X)_{D}^{I}\right| F$ satisfy the transfer principle. The following algebraic characterization for the internal models holds. A detailed proof can be found in $[\mathrm{D}]$.

Theorem 1.2 The mapping $\vartheta:\left\langle\mathrm{V}_{\alpha}(X), \in\right\rangle \rightarrow \mathcal{M}$ is a NSE if and only if there exist

- an ultrafilter $D$ over the set I

- a filter $F$ over the set $I \times I$

- an isomorphism $\pi:\left\langle\mathcal{I}_{\vartheta}, \epsilon\right\rangle \rightarrow{ }^{b} \mathrm{~V}_{\alpha}(X){ }_{D}^{I} \mid F$

such that the following diagram commutes:

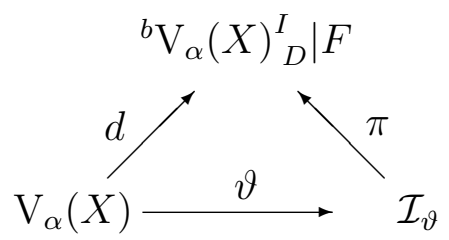

Moreover, the restrictions $\pi_{A} \doteq \pi\left\lceil\vartheta(A)_{\epsilon}:\left\langle\vartheta(A)_{\epsilon}, \epsilon\right\rangle \rightarrow A_{D}^{I} \mid F\right.$ are isomorphisms for every $A \in \mathrm{V}_{\alpha}(X)$.

This theorem is a straight generalization of a result proved by H.J. Keisler for $\alpha=\omega$ (see $[\mathrm{KE}] \S 1 . \mathrm{E}^{*}$ and $[\mathrm{CK}] \S 6.4$ ). It is now clear that in spite of nonwellfoundedness, the internal models retain an $\epsilon$-stratified structure. The next result will make this statement precise by showing that the internal models admit a rank function taking linearly ordered values. Let $\operatorname{Ord}(x, \varnothing)$ be the following bounded formula

$$
(x=\varnothing \vee \varnothing \in x) \wedge \text { " } x \text { is transitive" } \wedge \text { " } x \text { is linearly ordered by } \in \text { " }
$$

It is easily seen that 


$$
\mathrm{V}_{\alpha}(X) \models \operatorname{Ord}(\beta, \varnothing) \Longleftrightarrow \beta<\alpha \text { is a von Neumann ordinal }
$$

Call hyperordinal any $\xi \in \mathbf{M}$ such that $\mathcal{M}=\operatorname{Ord}(\xi, \vartheta(\varnothing))$, and consider $O r d_{\mathcal{I}}$ the collection of all the internal hyperordinals. If $\pi:\langle\mathcal{I}, \epsilon\rangle \rightarrow{ }^{b} \mathrm{~V}_{\alpha}(X){ }_{D}^{I} \mid F$ is the same isomorphism as in the previous theorem, then it is straightforwardly proved that the restriction

$$
\pi_{\mathcal{I}} \doteq \pi\left\lceil O r d_{\mathcal{I}}:\left\langle O r d_{\mathcal{I}}, \epsilon\right\rangle \longrightarrow{ }^{b} \alpha_{D}^{I}\left|F \doteq \bigcup_{\beta<\alpha} \beta_{D}^{I}\right| F\right.
$$

is an isomorphism as well. The set ${ }^{b} \alpha_{D}^{I} \mid F$ of the internal hyperordinals has some nice properties.

- ${ }^{b} \alpha_{D}^{I} \mid F$ is linearly ordered by the interpretation $\varepsilon$ of the membership relation symbol. For simplicity, we denote this ordering by $<$.

- Every $\xi \in{ }^{b} \alpha_{D}^{I} \mid F$ admits immediate successor $\xi+1$ where $(\xi+1)_{\varepsilon}=$ $\xi_{\varepsilon} \cup\{\xi\}$.

- Every nonempty internal collection of hyperordinals admits supremum.

As the diagonal embedding $d: \alpha \rightarrow{ }^{b} \alpha_{D}^{I} \mid F$ preserves the bounded formulas, all the above properties are straightforwardly proved by applying the transfer principle. Notice that the ordered set $\left\langle\operatorname{Ord}_{\mathcal{I}}, \epsilon\right\rangle \cong\left\langle{ }^{b} \alpha_{D}^{I} \mid F,<\right\rangle$ is neither wellordered nor order-complete. For instance, it is well-known that the external set $\Omega \doteq\{d(n): n<\omega\}$ lacks supremum and its complement lacks minimum in every nontrivial ultrapower $\alpha_{D}^{I}$ with $\alpha \geq \omega$.

Theorem 1.3 Let $\vartheta:\left\langle\mathrm{V}_{\alpha}(X), \in\right\rangle \rightarrow \mathcal{M}$ be a NSE and let $I, D$ and $F$ be the same as in the theorem 1.2. Then there exists a rank function $\psi_{\mathcal{I}}: \mathcal{I}_{\vartheta} \rightarrow$ ${ }^{b} \alpha_{D}^{I} \mid F$ for the internal submodel:

$$
\psi_{\mathcal{I}}(b)= \begin{cases}d(0) & \text { if } b \in \vartheta(X) \text { or } b=\vartheta(\varnothing) ; \\ \operatorname{Sup}\left\{\psi_{\mathcal{I}}\left(b^{\prime}\right)+1: b^{\prime} \in b\right\} & \text { otherwise. }\end{cases}
$$

Proof. For every $\beta<\alpha$ take the restriction $\rho_{\beta} \doteq \rho_{X}\left\lceil V_{\beta}(X): V_{\beta}(X) \rightarrow\right.$ $\beta+1$ of the rank function $\rho_{X}: \mathrm{V}_{\alpha}(X) \rightarrow \alpha . \rho_{\beta} \in \mathrm{V}_{\alpha}(X)$ and by applying the transfer principle to suitable bounded formulas, one gets a function $R_{\beta} \doteq$ $\left(\rho_{\beta}\right)_{\vartheta}: \vartheta\left(V_{\beta}(X)\right)_{\epsilon} \rightarrow \vartheta(\beta+1)_{\epsilon}$ such that

$$
R_{\beta}(b)= \begin{cases}\vartheta(0) & \text { if } b \in \vartheta(X) \text { or } b=\vartheta(\varnothing) \\ \operatorname{Sup}\left\{R_{\beta}\left(b^{\prime}\right)+1: b^{\prime} \epsilon b\right\} & \text { otherwise. }\end{cases}
$$


Notice that every collection $\left\{R_{\beta}\left(b^{\prime}\right)+1: b^{\prime} \epsilon b\right\}$ is internal. As $R_{\gamma}=R_{\beta}\left\lceil V_{\gamma}(X)\right.$ for every $\gamma<\beta<\alpha$, one can define $R_{\mathcal{I}}$ as the union $R_{\mathcal{I}} \doteq \bigcup_{\beta<\alpha} R_{\beta}$. Since $\bigcup_{\beta<\alpha} \vartheta\left(V_{\beta}(X)\right)_{\epsilon}=\mathcal{I}_{\vartheta}$ and $\bigcup_{\beta<\alpha} \vartheta(\beta)_{\epsilon}=O r d_{\mathcal{I}}$, it is easily seen that $\psi_{\mathcal{I}} \doteq$ $\pi_{\mathcal{I}} \circ R_{\mathcal{I}}$ satisfies the theses.

\section{The Pseudo-superstructures}

Though an algebraic characterization for the internal submodels is given by the Theorem 1.2, nevertheless a characterization for the full notion of nonstandard universe still lacks. In the author's opinion, a good notion of NSU should not be restricted to the internal sets. In fact, in the practice of nonstandard methods one continuously deals with external collections of internal sets, collections of collections of internal sets etc. E.g., in applications one uses such things as certain families of monads, or the $\sigma$-algebra generated by the algebra of internal subsets of a given set and so on. On the other hand, once all such subsets are added which are built up from the internal sets, nothing else is required. ${ }^{3}$ We claim that one can collapse the internal submodel into a transitive structure and then proceed in this filling up-way and obtain transitive classes which still are arranged in strata, in the likeness of the superstructures.

The main feature of the superstructures is their wellordered $\in$-stratification. Precisely one can characterize the $\alpha$-superstructures $\mathrm{V}_{\alpha}(X)$ as those sets $S$ which satisfy the following:

( $i$ ) $S$ is transitive up to atoms; ( $i i) S$ is provided with a rank function $\rho_{S}: S \rightarrow \alpha$ taking wellordered values ${ }^{4}$

$$
\rho_{S}(a)= \begin{cases}0 & \text { if } a \cap S=\varnothing \\ \operatorname{Sup}\left\{\rho_{S}\left(a^{\prime}\right)+1: a^{\prime} \in a\right\} & \text { otherwise }\end{cases}
$$

(iii) (Bounded universal property) If the set $A \subseteq S$ is rank-bounded (i.e. if $\left\{\rho_{S}(a): a \in A\right\} \subseteq \alpha$ is not cofinal) then $A \in S$. In particular $S$ is full.

In order to formulate the Representation Theorem for $\alpha$-NSU's, a straightforward generalization of the above properties to a nonwellfounded context is

\footnotetext{
${ }^{3}$ This (minimal) assumption will be formalized by the notion of "nonstandard universe which is wellfounded over the internal elements" (see the Representation Theorem 2.2).

${ }^{4}$ We will always assume a rank function to be onto.
} 
needed. Precisely, classes where the wellordered $\epsilon$-stratification is weakened to a linearly ordered $\in$-stratification will be considered. We give in fact the following

Definition A pseudo-superstructure (PSS) is a nonempty class $\mathbf{P}$ such that ( $i) \mathbf{P}$ is transitive up to atoms; ( $i i) \mathbf{P}$ is provided with a rank function $\psi_{P}: \mathbf{P} \rightarrow \lambda$ taking linearly ordered values

$$
\psi_{P}(p)= \begin{cases}0 & \text { if } p \cap \mathbf{P}=\varnothing ; \\ \operatorname{Sup}\left\{\psi_{P}\left(p^{\prime}\right)+1: p^{\prime} \in p\right\} & \text { otherwise. }\end{cases}
$$

(iii) (Bounded universal property) If $A \subseteq \mathbf{P}$ is a rank-bounded set (i.e. if $\left\{\psi_{P}(a): a \in A\right\} \subseteq \lambda$ is not cofinal) then $A \in \mathbf{P}$. In particular $\mathbf{P}$ is full.

We call $\mathbf{P} \alpha$-pseudo-superstructure $(\alpha$-PSS) if $\lambda$ includes a cofinal $\alpha$-sequence.

In the definition above it is implicitly assumed that $\lambda$ has minimum 0 and that every $\xi \in \lambda$ has immediate successor $\xi+1$. Moreover, though $\psi_{P}$ and $\lambda$ may be proper classes, the collections $\left\{\psi_{P}\left(p^{\prime}\right)+1: p^{\prime} \in p\right\}$ are sets for every $p \in \mathbf{P}$. Notice that $\lambda$ is upward complete in that every upper-bounded $\Xi \subset \lambda$ admits supremum (in fact, if $\Xi$ does not have a largest element, pick $p_{\xi} \in \mathbf{P}$ with $\psi_{P}\left(p_{\xi}\right)=\xi$ for each $\xi \in \Xi$ and let $p=\left\{p_{\xi}: \xi \in \Xi\right\}$. $p$ is rank-bounded and $\psi_{P}(p)=\operatorname{Sup}\left\{\psi_{P}\left(p_{\xi}\right)+1: \xi \in \Xi\right\}=\operatorname{Sup} \Xi$ exists by the bounded universal property). Notice also that for every $X$ which is $\alpha$-basal, $\mathrm{V}_{\alpha}(X)$ is trivially an $\alpha$-PSS. On the other hand, the Representation Theorem will show that PSS's exist which are not superstructures.

Let's first itemize the basic properties of PSS's. For every $\xi \in \lambda$, denote $\mathbf{P}_{\xi}=\left\{p \in \mathbf{P}: \psi_{P}(p) \leq \xi\right\}$. We point out that the $\mathbf{P}_{\xi}$ 's are in general proper classes. On the other hand, for convenience we suppose the collection of atoms $\mathbf{P}_{o} \backslash\{\varnothing\}$ to be a set.

Proposition 2.1 Let $\mathbf{P}$ be a PSS with the rank function $\psi_{P}: \mathbf{P} \rightarrow \lambda$. Then the following hold:

(i) $\mathbf{P}_{\xi+1}=\mathbf{P}_{\xi} \cup \mathcal{P}\left(\mathbf{P}_{\xi}\right)$ for every $\xi \in \lambda$.

(ii) $\langle\mathbf{P}, \in\rangle \models E X T_{o}, \varnothing, P A I R, S E P, U N, P S, A C$.

(iii) The next three properties are equivalent:

(a) $\lambda$ is wellordered; (b) $\langle\mathbf{P}, \in\rangle \models F O U N D$; (c) $\mathbf{P}=\mathrm{V}_{\alpha}(X)$ is an $\alpha$-superstructure with $X$-basal or $\mathbf{P}=\mathbf{W F}(X)$ with $X$ basal.

Now suppose that $\lambda$ is not wellordered. Then

(iv) $\mathbf{O N} \subset \mathbf{P}$. In particular $\langle\mathbf{P}, \in\rangle \models I N F$.

(v) $\langle\mathbf{P}, \in\rangle \models R E P \Leftrightarrow \mathbf{P}$ is not an $\alpha-P S S$ for any $\alpha$. 
Proof. (i) Apply the definition of rank and the bounded universal property. (ii) By the definition, the pseudo-superstructures $\mathbf{P}$ are nonempty, transitive up to atoms and full. Thus $\langle\mathbf{P}, \in\rangle \models E X T_{o}, \varnothing, S E P, U N, A C$. Moreover, many usual set-theoretic notions are absolute, i.e. they mean the same thing in $\mathbf{P}$ as in the universe. In particular, $\langle\mathbf{P}, \in\rangle \models P A I R, P S$ iff $\mathbf{P}$ is closed under the pairing and the power-set operators. Fix $p \in \mathbf{P}$. As $p^{\prime} \in p$ implies $\psi_{P}\left(p^{\prime}\right)<\psi_{P}(p)$, every $A \subseteq p$ is a rank-bounded set. Thus $A \in \mathbf{P}$ and $\psi_{P}(A) \leq \psi_{P}(p)+1$. In particular, $\mathcal{P}(p)$ is a rank-bounded set as well and $\mathcal{P}(p) \in \mathbf{P}$. Proceed similarly to prove $p, p^{\prime} \in \mathbf{P} \Rightarrow\left\{p, p^{\prime}\right\} \in \mathbf{P}$.

(iii) $(a) \rightarrow(b)$ and $(c) \rightarrow(a)$ are trivial. (b) $\rightarrow(a)$ Let $\xi_{o}>\xi_{1} \ldots>\xi_{n}>\ldots$ be a descending chain in $\lambda$. Take $p_{o} \in \mathbf{P}$ with $\psi_{P}\left(p_{o}\right)=\xi_{o}$. Then $\psi_{P}\left(p_{1}\right) \geq \xi_{1}$ for some $p_{1} \in p_{o}$ (otherwise $\psi_{P}(p)=\operatorname{Sup}\left\{\psi_{P}\left(p^{\prime}\right)+1: p^{\prime} \in p\right\} \leq \xi_{1}<\xi_{o}$, a contradiction). By iterating, one gets a descending chain $p_{o} \ni p_{1} \ni \ldots \ni$ $p_{n} \ni \ldots$ where $\psi_{P}\left(p_{n}\right) \geq \xi_{n}$ for every $n .\left\{p_{i}: i \in \omega\right\}$ is rank-bounded, hence in $\mathbf{P}$. This shows $\not \models F O U N D$. $(a) \wedge(b) \rightarrow(c)$ Assume $\lambda$ is wellordered and define by transfinite induction the functional correspondance:

$$
\mathbf{R}(0)=\operatorname{Min} \lambda ; \mathbf{R}(\alpha)=\operatorname{Sup}\{\mathbf{R}(\beta)+1: \beta<\alpha\} .
$$

$\operatorname{Domain}(\mathbf{R})$ is an initial segment of $\mathbf{O N}$ and $\operatorname{Range}(\mathbf{R})$ is an initial segment of $\lambda$ (proceed by transfinite induction on $\alpha$ and prove that $\xi<\mathbf{R}(\alpha) \rightarrow \xi \in$ Range $(\mathbf{R}))$. The following property holds:

"Suppose $A \in \mathbf{P}$. If $\psi_{P}(A) \notin$ Range $(\mathbf{R})$ then $\psi_{P}(a) \notin$ Range $(\mathbf{R})$ for some $a \in A$ ".

Otherwise, let $\psi_{P}(a)=\mathbf{R}\left(\beta_{a}\right)$ for every $a \in A$ and consider $\alpha=\operatorname{Sup}\left\{\beta_{a}\right.$ : $a \in A\}$. Notice that $\mathbf{R}(\beta)$ is defined for every $\beta<\alpha$, in that $\beta \leq \beta_{a} \in$ $\operatorname{Domain}(\mathbf{R})$ for some $\beta_{a}$. Then $\psi_{P}(A)=\operatorname{Sup}\left\{\psi_{P}(a)+1: a \in A\right\}=$ $\operatorname{Sup}\left\{\mathbf{R}\left(\beta_{a}\right)+1: a \in A\right\}=\operatorname{Sup}\{\mathbf{R}(\beta)+1: \beta<\alpha\}=\mathbf{R}(\alpha)$. As a straight consequence, one gets the equivalence $\langle\mathbf{P}, \in\rangle=F O U N D \Leftrightarrow \mathbf{R}$ is onto. Thus, let us suppose that $\mathbf{R}$ is onto. By transfinite induction, it is straightforwardly proved that $V_{\beta}\left(\mathbf{P}_{o}\right)=\mathbf{P}_{\beta}$ for every $\beta \in \operatorname{Domain}(\mathbf{R})$. In particular, if $X=\mathbf{P}_{o} \backslash\{\varnothing\}$ then $\mathbf{P}=\mathrm{V}_{\alpha}(X)$ or $\mathbf{P}=\mathbf{W F}(X)$ according to Range $(\mathbf{R})=\alpha$ or Range $(\mathbf{R})=\mathbf{O N}$ respectively.

(iv) Take $\xi_{o}>\xi_{1}>\ldots>\xi_{n}>\ldots$ a descending chain in $\lambda$. We prove by transfinite induction that every ordinal $\gamma \in \mathbf{P}$ and $\psi_{P}(\gamma)<\xi_{n}$ for every $n$. The case $\gamma=0$ is trivial. If $\gamma=\delta+1$, then $\langle\mathbf{P}, \in\rangle \models P A I R, U N$ and 
$\delta \in \mathbf{P}$ imply that $\delta \cup\{\delta\}=\gamma \in \mathbf{P}$. Besides, if $\psi_{P}(\gamma) \geq \xi_{n}$ for some $n$, then $\xi_{n+1}>\psi_{P}(\delta) \Rightarrow \xi_{n+1} \geq \psi_{P}(\delta)+1=\psi_{P}(\gamma) \geq \xi_{n}$, a contradiction. Now suppose $\gamma$ is limit. For every $\gamma^{\prime} \in \gamma$ and every $n, \psi_{P}\left(\gamma^{\prime}\right)<\xi_{n+1}$. As $\gamma \subset \mathbf{P}$ is a rank-bounded set, $\gamma \in \mathbf{P}$ and $\psi_{P}(\gamma)=\operatorname{Sup}\left\{\psi_{P}\left(\gamma^{\prime}\right)+1: \gamma^{\prime} \in \gamma\right\} \leq \xi_{n+1}<\xi_{n}$. (v) $\Rightarrow$ Let $\left\{\xi_{\beta}: \beta \in \alpha\right\} \subseteq \lambda$ be any $\alpha$-sequence. For each $\beta \in \alpha$, take $\mathbf{G}(\beta) \in \mathbf{P}$ such that $\psi_{P}(\mathbf{G}(\beta))=\xi_{\beta}$. By replacement, $\alpha \in \mathbf{P}$ implies $p=\{\mathbf{G}(\beta): \beta \in \alpha\} \in \mathbf{P}$. In particular, $\xi_{\beta}=\psi_{P}(\mathbf{G}(\beta))<\psi_{P}(p)$ for every $\beta \in \alpha$ and $\left\{\xi_{\beta}: \beta \in \alpha\right\}$ is not cofinal. $\Leftarrow$ Take a set $A \in \mathbf{P}$ and let $\mathbf{G}$ be a functional correspondance such that $\mathbf{G}(a) \in \mathbf{P}$ when $a \in A$. Then $\{\mathbf{G}(a): a \in A\} \in \mathbf{P}$ because it is a rank-bounded subset of $\mathbf{P}$.

Notice that property (i) is the counterpart of the usual inductive definition of superstructure. Thus, in close analogy to the cumulative hierarchy one can write

$$
\mathbf{P}=\bigcup_{\xi \in \lambda} \mathbf{P}_{\xi} \quad \text { where } \mathbf{P}_{\xi+1}=\mathbf{P}_{\xi} \cup \mathcal{P}\left(\mathbf{P}_{\xi}\right) .
$$

As the following theorem shows, the notion of PSS is well suited for characterizing the $\alpha$-NSU's. Without loss of generality, in the following we suppose $X$ of the same form as in the example of Section 1 (recall that such basal sets exist of any size).

\section{Theorem 2.2 THE REPRESENTATION THEOREM}

Let $\left\langle\mathrm{V}_{\alpha}(X), \in\right\rangle$ be a standard $\alpha$-model and suppose that $\vartheta:\left\langle\mathrm{V}_{\alpha}(X), \in\right\rangle \rightarrow$ $\langle\mathbf{M}, \epsilon\rangle$ is a nonstandard embedding. Then there exist

- an $\alpha$-pseudo-superstructure $\langle\mathbf{P}, \in\rangle$ with rank $\psi_{P}: \mathbf{P} \rightarrow \lambda$

- a nonstandard embedding $*:\left\langle\mathrm{V}_{\alpha}(X), \in\right\rangle \rightarrow\langle\mathbf{P}, \in\rangle$

- an isomorphism $\tau:\left\langle\mathcal{I}_{\vartheta}, \epsilon\right\rangle \rightarrow\left\langle\mathcal{I}_{*}, \in\right\rangle$ between the internal submodels relative to $\vartheta$ and $*$ respectively

such that

(i) $\mathbf{P}$ is wellfounded over the internal elements, i.e. no descending chain $b_{1} \ni b_{2} \ni \ldots \ni b_{n} \ni \ldots$ exists in $\mathbf{P}$ where all the $b_{n}$ 's are external.

$($ ii $){ }^{*} X$ is the set of atoms relative to $\langle\mathbf{P}, \in\rangle$.

(iii) ${ }^{*} x=x$ for every $x \in X$

(iv) $\psi_{P}\left({ }^{*} A\right)={ }^{*} \rho_{X}(A)$ for every $A \in \mathrm{V}_{\alpha}(X)$. 
(v) $\left\{\psi_{P}\left({ }^{*} \beta\right): \beta \in \alpha\right\} \subseteq \lambda$ is an increasing cofinal $\alpha$-sequence.

(vi) the following diagram commutes:

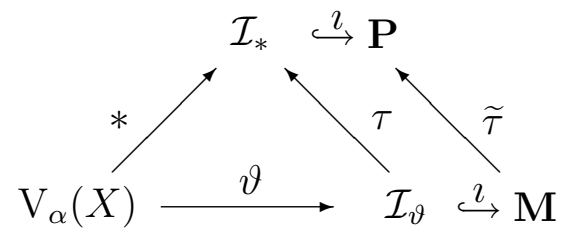

Moreover, if $\langle\mathbf{M}, \epsilon\rangle$ is wellfounded over the internal elements, $\tau$ can be extended in a natural way so as to get an isomorphic embedding $\widetilde{\tau}: \mathbf{M} \rightarrow \mathbf{P}$.

Proof. Take the ultrafilter $D$ over the set $I$, the filter $F$ over $I \times I$ and the isomorphism $\pi:\left\langle\mathcal{I}_{\vartheta}, \epsilon\right\rangle \rightarrow{ }^{b} \mathrm{~V}_{\alpha}(X)_{D}^{I} \mid F$ as given by the Thereom 1.2. Recall that $X=\left\{x_{j}: j \in \mu\right\}$ where $x_{j}=\left\{y_{j}\right\}, y_{j}=\left\{x_{j}, y_{j}\right\}$ and $x_{j} \neq y_{j^{\prime}}$ for every $j, j^{\prime} \in \mu$, and denote $Y=\left\{y_{j}: j \in \mu\right\}$. Let $d$ be the diagonal embedding of $V_{\alpha}(X \cup Y)$ into the bounded limit ultrapower ${ }^{b} V_{\alpha}(X \cup Y)_{D}^{I} \mid F$ and denote $\Lambda=\{d(z): z \in X \cup Y \cup\{\varnothing\}\}$. As every $z \in X \cup Y$ is a finite set, $\left\langle{ }^{b} V_{\alpha}(X \cup Y){ }_{D}^{I} \mid F, \varepsilon\right\rangle$ is an end extension of $\langle\Lambda, \varepsilon\rangle$. Moreover, by the transitivity of $X \cup Y$ and the elementary embedding property of $d$ one gets that the both structures are extensional. Thus the axiom $S U$ can be applied so as to get an isomorphism

$$
\sigma:{ }^{b} V_{\alpha}(X \cup Y){ }_{D}^{I} \mid F \rightarrow\langle S, \in\rangle
$$

onto a transitive set $S$. As the mapping $d(z) \mapsto z$ yields an isomorphism $\langle\Lambda, \varepsilon\rangle \cong\langle X \cup Y \cup\{\varnothing\}, \in\rangle, \sigma$ can be taken is such a way that $\sigma(d(z))=z$ for every $z \in X \cup Y \cup\{\varnothing\}$. We now eliminate everything which is grounded over $Y$. Precisely, we consider the restriction $\tau=\sigma \Gamma^{b} \mathrm{~V}_{\alpha}(X)_{D}^{I} \mid F$ which is an isomorphism

$$
\tau:{ }^{b} \mathrm{~V}_{\alpha}(X)_{D}^{I} \mid F \rightarrow\langle T, \in\rangle
$$

onto the set $T=\operatorname{Range}(\tau) \subset S$. Notice that $T$ is transitive up to atoms. Our goal is making an $\alpha$-PSS out of $T$. Let $\psi_{T} \doteq \psi_{\mathcal{I}} \circ \pi^{-1} \circ \tau^{-1}$ be the rank function for $T$ inherited from the internal model $\mathcal{I}^{5}$ and denote $T_{\beta} \doteq$ $\left\{t \in T: \psi_{T}(t) \leq d(\beta)\right\}$ for every $\beta \in \alpha$. The peculiar $\in$-structure of $X$ and $Y$ is reflected in $\left\langle(X \cup Y)_{D}^{I} \mid F, \varepsilon\right\rangle$ and the following property holds: "if

\footnotetext{
${ }^{5}$ See the theorems 1.2 and 1.3 .
} 
$f^{D} \in X_{D}^{I} \mid F$ then $f_{\varepsilon}^{D}=\left\{g^{D}\right\}$ and $g_{\varepsilon}^{D}=\left\{f^{D}, g^{D}\right\}$ for some $g^{D} \in Y_{D}^{I} \mid F^{\prime \prime}$. Notice that $T_{o}=\left\{\tau\left(f^{D}\right): f^{D} \in X_{D}^{I} \mid F\right\} \cup\{\varnothing\}$ and $Y_{D}^{I}\left|F \cap X_{D}^{I}\right| F=\varnothing$. Thus

- Every $\varnothing \neq t \in T_{o}$ has the form $t=\{w\}$, where $w=\{t, w\} \notin T_{o}$.

In order to get the bounded universal property, we fill up $T$ with subsets. Precisely we define

$$
\mathbf{P}=\bigcup_{\beta \in \alpha} \mathbf{W F}\left(T_{\beta}\right)
$$

If $A \in \mathbf{P}$ is $\in$-minimal then trivially $A \in T_{o}$. Vice versa, suppose $t=\{w\} \in$ $T_{o}$ and $t \cap \mathbf{P} \neq \varnothing$. Then $w=\{t, w\} \in \mathbf{P}$ for some $w \notin T_{o}$. This is a contradiction because it is straightforwardly proved that $w \notin T_{\beta}$ for every $\beta<\alpha$ and that $w \notin V_{\gamma}\left(T_{\beta}\right)$ for every $\gamma$. Thus the following holds:

- $p \in \mathbf{P}$ and $p \cap \mathbf{P}=\varnothing \Leftrightarrow p \in T_{o}$, i.e. $\langle\mathbf{P}, \in\rangle$ has the same set of atoms as $\langle T, \in\rangle$.

We claim that extending $\psi_{T}$ to a rank function $\psi_{P}$ for $\mathbf{P}$ is possible. To this end, consider the order-completion $\lambda^{\prime}$ of $\zeta=\left\langle{ }^{b} \alpha_{D}^{I} \mid F, \varepsilon\right\rangle$ and replace every new element $x \in \lambda^{\prime} \backslash \zeta$ with a copy $\mathbf{O N}_{x}$ of the ordinals so as to get the linearly ordered proper class $\lambda \supset \lambda^{\prime} \supset \zeta$ (we identify $x$ with $0_{x}$ ). Notice that $\{d(\beta): \beta \in \alpha\} \subset \lambda$ is an increasing cofinal $\alpha$-sequence. For every $\beta \in \alpha$, define $\psi_{\beta}: \mathbf{W F}\left(T_{\beta}\right) \rightarrow \lambda$ as follows

$$
\psi_{\beta}(A)= \begin{cases}\psi_{T}(A) & \text { if } A \in T_{\beta} \\ \operatorname{Sup}\left\{\psi_{\beta}(a)+1: a \in A\right\} & \text { otherwise. }\end{cases}
$$

Denote $\lambda^{(\gamma)} \doteq \lambda^{\prime} \cup\left\{\left\{0_{x}, 1_{x}, \ldots, \gamma_{x}\right\}: x \in \lambda^{\prime} \backslash \zeta\right\}$ the subset of $\lambda$ where only the initial segments of lenght $\gamma$ are included of the various $\mathbf{O N}_{x}$ 's. By transfinite induction on $\gamma$ one proves the following facts:

- $A \in V_{\gamma+1}\left(T_{\beta}\right) \Rightarrow \psi_{\beta}(A) \in \lambda^{(\gamma)}$

- $A \in V_{\gamma}\left(T_{\beta}\right) \cap T \Rightarrow \psi_{\beta}(A)=\psi_{T}(A)$

Thus $\psi_{\beta}$ is well-defined and $\psi_{\beta}(A)=\psi_{T}(A)$ whenever $A \in \mathbf{W F}\left(T_{\beta}\right) \cap T$. Given $\beta<\beta^{\prime}<\alpha$, again proceeding by transfinite induction on $\gamma$, one also proves that 
- $\psi_{\beta}(A)=\psi_{\beta^{\prime}}(A)$ for every $A \in V_{\gamma}\left(T_{\beta}\right)$

and the following is well-defined as well

$$
\psi_{P} \doteq \bigcup_{\beta \in \alpha} \psi_{\beta}: \mathbf{P} \rightarrow \lambda
$$

$\psi_{P}$ is a rank for $\langle\mathbf{P}, \in\rangle$. In fact $p \in \mathbf{P}$ is $\in$-minimal iff $p \in T_{o}$ iff $\psi_{P}(p)=d(0)$. If $p \in \mathbf{P}$ is not $\in$-minimal, the property $\psi_{P}(p)=\operatorname{Sup}\left\{\psi\left(p^{\prime}\right)+1: p^{\prime} \in p\right\}$ straightforwardly follows from the definition of the $\psi_{\beta}$ 's. $\mathbf{P}$ satisfies the bounded universal property (iii) in the definition of PSS. In fact, let $A \subseteq \mathbf{P}$ be any set and define $\beta_{a}=\operatorname{Min}\left\{\beta \in \alpha: a \in \mathbf{W F}\left(T_{\beta}\right)\right\}$ and $\gamma_{a}=\operatorname{Min}\{\gamma \in$ ON : $\left.a \in V_{\gamma}\left(T_{\beta_{a}}\right)\right\}$ for each $a \in A$. As $A$ is rank-bounded, $\beta=\operatorname{Sup}\left\{\beta_{a}: a \in\right.$ $A\}<\alpha$ and so $A \in V_{\gamma+1}\left(T_{\beta}\right) \subseteq \mathbf{P}$ where $\gamma=\operatorname{Sup}\left\{\gamma_{a}: a \in A\right\}$. T and $\mathbf{P}$ are transitive up to atoms, thus the bounded formulas are preserved by the inclusion map $\imath: T \hookrightarrow \mathbf{P}$ and the composition

$$
*=\imath \circ \tau \circ \vartheta:\left\langle\mathrm{V}_{\alpha}(X), \in\right\rangle \longrightarrow\langle\mathbf{P}, \in\rangle
$$

is a NSE. The internal submodel $\mathcal{I}_{*}$ relative to $*$ is easily shown to be the set $T$, thus $\tau$ is an isomorphism between the internal submodels $\left\langle\mathcal{I}_{\vartheta}, \epsilon\right\rangle$ and $\left\langle\mathcal{I}_{*}, \in\right\rangle$. As for the properties (i), (ii), (iii), (v) and (vi), they are all proved by looking at the definitions of the sets and functions involved. Property (iv) holds provided that one identifies $\zeta=\left\langle{ }^{b} \alpha_{D}^{I} \mid F, \varepsilon\right\rangle \subseteq \lambda$ with its isomorphic copy $\left\{\tau\left(\pi^{-1}(\xi)\right): \xi \in \lambda\right\} \subseteq \mathbf{P} .{ }^{6}$ Now suppose that $\langle\mathbf{M}, \epsilon\rangle$ is wellfounded over $\mathcal{I}_{\vartheta}$. Then it is easily shown by $\epsilon$-induction that

$$
\widetilde{\tau}(m)= \begin{cases}\tau(m) & \text { if } m \in \mathcal{I}_{\vartheta} \\ \left\{\widetilde{\tau}\left(m^{\prime}\right): m^{\prime} \in m\right\} & \text { otherwise }\end{cases}
$$

is well-defined and provides an isomorphic embedding of $\mathbf{M}$ in $\mathbf{P} . \quad-$

The pseudo-superstructures succeed in meeting all the basic requirements for a general notion of nonstandard $\alpha$-universe. In fact, compare the following properties with properties $1,2,3$ and 4 itemized in the first section for $\omega$ NSU's.

1. Every internal model can be isomorphically collapsed onto an $\in$-structure which is transitive up to atoms.

\footnotetext{
${ }^{6} \pi$ is the same as in the Theorem 1.2 .
} 
2. Up to isomorphisms, every internal model is the internal model of a pseudo-superstructure and every nonstandard universe which is wellfounded over the internal elements is a submodel of a pseudo-superstructure.

3. Pseudo-superstructures are transitive up to atoms and full.

4. Pseudo-superstructures are linearly founded, in that they admit a rank function taking linearly ordered values.

Consequently, without loss of generality one can only consider $\alpha$-PSS's as $\alpha$-NSU's and propose the following

Definition A nonstandard $\alpha$-universe relative to the standard $\alpha$-model $\left\langle\mathrm{V}_{\alpha}(X), \in\right\rangle$ is any $\alpha$-PSS $\left\langle\mathbf{P}, \psi_{P}\right\rangle$ equipped with a mapping $*: \mathrm{V}_{\alpha}(X) \rightarrow \mathbf{P}$ in such a way that the following properties hold:

(i) * satisfies the transfer principle for every bounded formula.

(ii) ${ }^{*} X$ is the set of atoms relative to $\langle\mathbf{P}, \in\rangle$.

(iii) ${ }^{*} x=x$ for every $x \in X$.

(iv) $\psi_{P}\left({ }^{*} A\right)={ }^{*} \rho_{X}(A)$ for every $A \in \mathrm{V}_{\alpha}(X)$.

(v) $\left\{\psi_{P}\left({ }^{*} \beta\right): \beta \in \alpha\right\} \subseteq \lambda$ is an increasing cofinal $\alpha$-sequence.

(vi) For every infinite set $A \in \mathrm{V}_{\alpha}(X),\left\{{ }^{*} a: a \in A\right\} \neq *^{*} A$.

(vii) $\mathbf{P}$ is wellfounded over the internal elements.

\section{Pseudo-superstructures for nonstandard set theory}

As shown by the previous theorem, the $\alpha$-PSS's characterize the notion of nonstandard $\alpha$-universe. The next result shows that the notion of PSS is well suited also for the whole set theory, and allows all the useful properties commonly used in the practice of nonstandard methods. The proof is obtained by combining our construction in the proof of the Representation Theorem 2.2. with arguments in $[\mathrm{BH}]$, where a global form of the axiom of choice is assumed.

Theorem 3.1 Let $\mu, \kappa$ be given cardinals. Then there exists a nonstandard embedding $*: \mathcal{S} \rightarrow \mathbf{P}$ into a pseudo-superstructure $\mathbf{P}$ such that: 
(i) $\mathcal{S}=\langle\mathbf{W F}(X), \in\rangle \models Z F C_{o}$ is the wellfounded universe over a set $X$ of atoms of power $\mu ;{ }^{7}$

(ii) $\mathbf{P}$ satisfies the following strong fullness property: " $A \subseteq \mathbf{P} \Rightarrow A \in \mathbf{P}$ for every set $A "$;

(iii) $\langle\mathbf{P}, \in\rangle \models Z F C_{o}^{-}$;

(iv) $*: \mathcal{S} \rightarrow \mathcal{I}$ is an elementary embedding into the internal model;

(v) * satisfies the $\kappa^{+}$-saturation property, i.e. if $\mathcal{F}$ is a family of internal sets, $|\mathcal{F}| \leq \kappa$ and $\cap \mathcal{F}_{o} \neq \varnothing$ for every finite $\mathcal{F}_{o} \subseteq \mathcal{F}$, then $\cap \mathcal{F} \neq \varnothing$;

(vi) No descending chain $b_{1} \ni b_{2} \ni \ldots \ni b_{n} \ni \ldots$ exists in $\mathbf{P}$ where all the $b_{n}$ 's are external.

Proof. Let $X=\left\{x_{j}: j \in \mu\right\}$ be a basal set of cardinality $\mu$ where (as usual) $x_{j}=\left\{y_{j}\right\}, y_{j}=\left\{x_{j}, y_{j}\right\}$, and $x_{j} \neq y_{j^{\prime}}$ for every $j, j^{\prime} \in \mu$. The transitive closure of $X$ is $X \cup Y$ with $Y=\left\{y_{j}: j \in \mu\right\}$. In particular $\langle X \cup Y, \in\rangle$ is extensional. The next arguments are a slight modification of the construction given in the proof of the Extension Principle of $[\mathrm{BH}]$. Thus, we sketch it in five steps and refer the reader to that paper for the details.

1. Take $D$ a $\kappa^{+}$-good countably incomplete ultrafilter over $\kappa$ and consider the diagonal embeddings $d_{\alpha}: \mathrm{V}_{\alpha}(X) \rightarrow V_{\alpha}(X \cup Y)^{\kappa}{ }^{\kappa}{ }^{8}$

2. For every $\beta<\alpha$, the extensional structure $V_{\alpha}(X \cup Y)_{D}^{\kappa}$ is an end extension of $V_{\beta}(X \cup Y)_{D}^{\kappa}$.

3. Using transfinite recursion and the axiom $S U$, one gets isomorphisms $\sigma_{\alpha}: V_{\alpha}(X \cup Y)_{D}^{\kappa} \rightarrow\left\langle S_{\alpha}, \in\right\rangle$ in such a way that $\sigma_{\alpha}\left\lceil V_{\beta}(X \cup Y)_{D}^{\kappa}=\sigma_{\beta}\right.$ for every $\beta<\alpha$. Moreover, one can take $\sigma_{\alpha}(z)=z$ for every $z \in X \cup Y$. 9

4. The restrictions $\sigma_{\alpha}\left\lceil\mathrm{V}_{\alpha}(X)_{D}^{\kappa}\right.$ yield isomorphisms $\tau_{\alpha}: \mathrm{V}_{\alpha}(X)_{D}^{\kappa} \rightarrow\left\langle T_{\alpha}, \in\right.$ $>$ onto sets $T_{\alpha}$ which are transitive up to atoms.

${ }^{7} Z F C_{o}$ denotes $Z F C$ where the extensionality axiom $E X T$ is replaced by $\mathrm{EXT}_{o}$.

${ }^{8}$ Recall the following theorems of Keisler. 1) "Let I be a set of power $\nu$. Then there exists a $\nu^{+}$-good countably incomplete ultrafilter over I". 2) "Let $D$ be a $\nu$-good countably incomplete ultrafilter over $I$ and let $\mathcal{A}$ be a model for the language $\mathcal{L}$ with $\|\mathcal{L}\|<\nu$. Then the ultrapower $\mathcal{A}_{D}^{I}$ is $\nu$-saturated". (See $[\mathrm{CK}] \S 6.1$ ).

${ }^{9}$ See the proof of the Theorem 2.2. In order to make the correspondance $\alpha \mapsto \sigma_{\alpha}$ definable, one takes $\sigma_{\alpha}$ as the least isomorphism (in the well-ordering of the universe) having the desired properties. 
5. Consider the mapping $\mathbf{F}: \mathbf{W F}(X) \rightarrow \mathbf{T}$ where $\mathbf{T}=\bigcup_{\alpha} T_{\alpha}$ and $\mathbf{F}=$ $\bigcup_{\alpha} \tau_{\alpha} \circ d_{\alpha}$. Simultaneous reflection principles hold for $\operatorname{both}\langle\mathbf{W F}(X), \in\rangle$ and $\langle\mathbf{T}, \in\rangle$, thus $\mathbf{F}$ is an elementary embedding of $\in$-structures.

Now let $\mathcal{S}=\langle\mathbf{W F}(X), \in\rangle, \mathbf{P}=\bigcup_{\alpha} \mathbf{W F}\left(T_{\alpha}\right)$ and define

$$
* \doteq \imath \circ \mathbf{F}: \mathbf{W F}(X) \longrightarrow \mathbf{P}
$$

* satisfies the transfer principle, because $\mathbf{F}$ is an elementary embedding and $\imath$ is an inclusion of classes which are transitive up to atoms. Notice that the internal submodel $\mathcal{I}_{*}=\mathbf{T} \cong \bigcup_{\alpha} \mathrm{V}_{\alpha}(X)_{D}^{\kappa}$. Thus the property (iv) is given by 5 . and the saturation property $(v)$ holds because $D$ is $\kappa^{+}$-good. Properties (i) and (vi) straightforwardly follow from the definitions.

Now let's turn to the rank function and prove (ii) and (iii). For each $\alpha$, consider the rank function $\psi_{\alpha}: \mathbf{W F}\left(T_{\alpha}\right) \rightarrow \lambda_{\alpha}$ as defined in the proof of the Representation Theorem 2.2. Without loss of generality, we can suppose $\lambda_{\beta} \subset \lambda_{\alpha}$ for every $\beta<\alpha$. In fact, the definition of each $\lambda_{\alpha}$ is based on the order-completion of $\left\langle{ }^{b} \alpha_{D}^{\kappa}, \epsilon\right\rangle$ which is in turn defined by means of the usual Dedekind cut construction. Thus one can take the unions $\psi_{P}=\bigcup_{\alpha} \psi_{\alpha}$ and $\lambda=\bigcup_{\alpha} \lambda_{\alpha}$ and get a rank function $\psi_{P}: \mathbf{P} \rightarrow \lambda$ where $\left\{\psi_{P}\left({ }^{*} \alpha\right): \alpha \in \mathbf{O N}\right\}$ is a cofinal subclass of $\lambda$. Let $A$ be any set with $A \subseteq \mathbf{P}$. For each $a \in A$ one can take $\beta_{a} \in \mathrm{ON}$ with $\psi_{P}(a) \leq \psi\left({ }^{*} \beta_{a}\right)$ and consider $\beta=S u p\left\{\beta_{\alpha}: a \in A\right\}$. Then $A$ is rank-bounded by $\psi_{P}\left({ }^{*} \beta\right)$ and $A \in \mathbf{P}$. This completes the proof of (ii). Now look at the Proposition 2.1. As $\lambda$ is not wellordered and is not cofinal with any $\alpha$-sequence, one gets that $\langle\mathbf{P}, \in\rangle \models Z F C_{o}^{-}$.

We summarize the main features of our construction.

- Nonstandard methods are allowed with respect to the whole standard ZFC mathematics.

- All usual standard $Z F C^{-}$arguments can be performed in the nonstandard universes, which are transitive up to atoms and (strongly) full classes.

- Though nonwellfounded, the nonstandard universes $\mathbf{P}$ are provided with a rank function $\psi_{P}(p)=\operatorname{Sup}\left\{\psi_{P}\left(p^{\prime}\right)+1: p^{\prime} \in p\right\}$ taking linearly ordered values. 
- In the likeness of the superstructures, the nonstandard universes $\mathbf{P}$ have a cumulative hierarchy structure

$$
\mathbf{P}=\bigcup_{\xi \in \lambda} \mathbf{P}_{\xi} \text { where } \mathbf{P}_{\xi+1}=\mathbf{P}_{\xi} \cup \mathcal{P}\left(\mathbf{P}_{\xi}\right)
$$

- The external elements are wellfounded with respect to to the internal sets, i.e. no descending chain

$$
b_{1} \ni b_{2} \ni \ldots \ni b_{n} \ni \ldots
$$

exists where all the $b_{n}$ 's are external.

- The $\kappa$-saturation property holds.

- The full standardization property is satisfied: "for every $A$, there exists a standard set $B$ such that $A$ and $B$ have the same standard elements" (take $B={ }^{*} A^{\prime}$ where $A^{\prime}=\left\{x \in \mathcal{S}:{ }^{*} x \in A\right\}$ ).

Acknowledgments. The author wishes to thank Marco Forti for many useful discussions on the subject and wishes to express his deep gratitude to Karel Hrbacek for his helpful remarks on preliminary drafts of this paper.

\section{References}

[A] P. ACzEL, Non-Well-Founded Sets, CSLI Lecture Notes, vol. 14, Center for the Study of Language and Information, Stanford, California, 1988.

[AR] P. America and J.J.M.M. Rutten, Solving Domain Equations in a Category of Complete Metric Spaces, J. Comp. Sys. Sci., vol. 39 (1989), pp. 343-375.

[B] M. Boffa, Forcing et Négation de l'Axiome de Fondement, Mem. Acad. Sc. Belg., Tome XL, no. 7 (1972).

[BH] D. Ballard and K. HrbaceK, Standard Foundations for Nonstandard Analysis, J. Symb. Logic, vol. 57 (1992), pp. 741-748. 
[CK] C.C. Chang and H.J. KeIsler, Model Theory, 3rd edition, North-Holland, Amsterdam, 1990 (1st edition 1973).

[D] M. Di NAsso, Hyperordinals and Nonstandard $\alpha$-Models, in Logic and Algebra (A. Ursini and P. Aglianò, eds.), Lecture Notes in Pure and Applied Mathematics, vol. 180, Dekker, New York, 1996, pp. 457-475.

[Fu] P. Fletcher, Nonstandard Set Theory, J. Symb. Logic, vol. 54 (1989), pp. 1000-1008.

[FH] M. Forti and F. Honsell, Set Theory with Free Construction Principles, Ann. Scuola Norm. Sup. Pisa, Cl. Sci. (IV), vol. 10 (1983), pp. 493-522.

[Hi] R. Hinnion ed., L'Anti-fondation en Logique et en Theorie des Ensembles, Cahiers du Centre de Logique, vol. 7, Louvain-laNeuve, 1992.

[Hr] K. HrbaceK, Nonstandard Set Theory, Am. Math. Monthly, vol. 86 (1979), pp. 659-677.

[J] T. Jech, Set Theory, Academic Press, New York, 1978.

[KA] T. KawAI, Nonstandard Analysis by Axiomatic Method, in Southeast Asian Conference on Logic (proceedings), Singapore 1981 (C.T. Chong and M.J. Wicks, eds.), Studies in Logic and the Foundations of Mathematics, vol. 111, North-Holland, Amsterdam, 1983, pp. 55-76.

[Ke] H.J. KeIsler, Foundations of Infinitesimal Calculus, Prindle, Weber \& Schmidt, Boston, 1976.

[N] E. Nelson, Internal Set Theory: a New Approach to Nonstandard Analysis, Bull. of the Am. Math. Soc., vol. 83 (1977), pp. 11651198.

[RZ] A. Robinson and E. ZAKon, A Set-Theoretical Characterization of Enlargements, in Applications of Model Theory to Algebra, Analysis and Probability (W.A.J. Luxemburg, ed.), Holt, Rinehart and Winston, New York, 1969, pp. 109-122. 
[SS] J. Schmid and J. SchmidT, Enlargements without Urelements, Colloq. Math., vol. 52 (1987), pp. 1-22.

[Z] E. ZAKOn, A New Variant of Nonstandard Analysis, in Victoria Symposium on Nonstandard Analysis (A.E. Hurd and P.A. Loeb, eds.), Lecture Notes in Mathematics, vol. 369, Springer-Verlag, Berlin, 1974, pp. 313-339.

Dipartimento Di MATEMATICA, UNIVERSITÀ DI SIENA.

Via del CAPitano, 15.

53100 SiEnA, ITALY.

E-MAIL: dinasso@unisi.it 